\title{
On the number of vertices of the stochastic tensor polytope*
}

\author{
Zhongshan $\mathrm{Li}^{\mathrm{a}}$, Fuzhen Zhang ${ }^{\mathrm{b}}$, Xiao-Dong Zhang ${ }^{\mathrm{c}}$ \\ a Georgia State University, Atlanta, USA; zli@gsu.edu \\ b Nova Southeastern University, Fort Lauderdale, USA; zhang@nova.edu \\ c Shanghai Jiao Tong University, Shanghai, China; xiaodong@sjtu.edu.cn
}

In memory of Professor Marvin Marcus (1927-2016).

\begin{abstract}
This paper is devoted to the study of lower and upper bounds for the number of vertices of the polytope of $n \times n \times n$ stochastic tensors (i.e., triply stochastic arrays of dimension $n$ ). By using known results on polytopes (i.e., the Upper and Lower Bound Theorems), we present some new lower and upper bounds. We show that the new upper bound is tighter than the one recently obtained by Chang, Paksoy and Zhang [Ann. Funct. Anal. 7 (2016), no. 3, 386-393] and also sharper than the one in Linial and Luria's [Discrete Comput. Geom. 51 (2014), no. 1, 161-170]. We demonstrate that the analog of the lower bound obtained in such a way, however, is no better than the existing ones.
\end{abstract}

AMS Classification: Primary 15B51; Secondary 52B11.

Keywords: Birkhoff polytope, Birkhoff-von Neumann theorem, doubly stochastic matrix, extreme point, hypermatrix, multidimensional matrix, polytope, stochastic semi-magic cube, stochastic tensor, vertex.

\section{Introduction}

Recall the well-known Birkhoff polytope $\omega_{n}$ of $n \times n$ doubly stochastic matrices: $\omega_{n}$ is the convex hull of all $n \times n$ permutation matrices. As a polytope in $\mathbb{R}^{n^{2}}$, $\omega_{n}$ has dimension $(n-1)^{2}$ with $n^{2}$ facets, and $n$ ! vertices.

Consider multi-arrays of higher dimension (here we focus on 3rd order). By a stochastic tensor (or cube) of dimension $n$ we mean a real $n \times n \times n$ hypermatrix (a.k.a. 3rd order tensor of dimension $n) A=\left(a_{i j k}\right)$ satisfying the conditions:

$$
\begin{gathered}
a_{i j k} \geq 0, \quad 1 \leq i, j, k \leq n \\
\sum_{i=1}^{n} a_{i j k}=1, \quad 1 \leq j, k \leq n
\end{gathered}
$$

\footnotetext{
*To appear in Linear and Multilinear Algebra special issue dedicated to M. Marcus.
} 


$$
\begin{aligned}
& \sum_{j=1}^{n} a_{i j k}=1, \quad 1 \leq i, k \leq n \\
& \sum_{k=1}^{n} a_{i j k}=1, \quad 1 \leq i, j \leq n
\end{aligned}
$$

Let $\Omega_{n}$ be the set of all $n \times n \times n$ stochastic tensors. Consider each $A \in \Omega_{n}$ as an element of $\mathbb{R}^{n^{3}}$. Then $\Omega_{n}$ is a subset of $\mathbb{R}^{n^{3}}$. Because it is an intersection of a finite number of closed half spaces (as a linear equation $A x=b$ is equivalent to $A x \leq b$ and $A x \geq b$ ) and it is bounded, $\Omega_{n}$ is a polytope; that is, it is generated by (or a convex hull of) finitely many points, i.e., extreme points or vertices of $\Omega_{n}$. 0-1 permutation tensors (i.e., stochastic tensors with 0 and 1 entries) are vertices of the polytope, but there are others in general (when $n>2$ ). For the case of $\Omega_{3}$, it is known that there are 12 0-1 permutation tensors as vertices and 54 other vertices that are non 0-1 (see [2, p. 34]). For a general $n$, the number of $0-1$ permutation tensors of order 3 and dimension $n$ is the same as the number of $n \times n$ Latin squares (see [17, p. 159] or [9, Proposation 2.6]).

Note that the Birkhoff-von Neumann theorem for multistochastic tensors is investigated by Cui, $\mathrm{Li}$ and $\mathrm{Ng}$ [9], Fischer and Swart [12], etc., and that a similar polytope of polystochastic matrices has been studied by Gromova 13. (see also [4, p. 64]), and by Brualdi and Csima [6, 7]. To our knowledge, Juflkat and Ryser 14 are the first ones who directly addressed and studied the multidimensional matrices which now we call tensors (while general tensors have broader meanings depending contexts).

Although $\Omega_{n}$ is an analog of $\omega_{n}$ for higher order, $\Omega_{n}$ has many different geometric properties than $\omega_{n}$. The determination of the number and structures of the vertices of $\Omega_{n}$ is a very difficult problem. Estimation of the number of vertices has been witnessed in three ways: (1). Combinatorial method via Latin squares. Ahmed, De Loera, and Hemmecke (see [2, Theorem 0.1] or [1. Theorem 2.0.10]) gave an explicit lower bound $\frac{(n !)^{2 n}}{n^{n^{2}}}$. (2). Analytic (and direct) approach by using hyperplane and induction. Chang, Paksoy and Zhang [8] recently showed an upper bound (see Theorem 1 below). (3). Computational geometry approach using known results on polytopes. Adopting this approach, we in this paper present some upper and lower bounds of the number of vertices of $\Omega_{n}$ and compare the new bounds with the existing ones.

Theorem 1 Let $f_{0}\left(\Omega_{n}\right)$ be the number of vertices of the polytope $\Omega_{n}$. Then

$$
\frac{(n !)^{2 n}}{n^{n^{2}}} \leq f_{0}\left(\Omega_{n}\right) \leq \frac{1}{n^{3}} \cdot\left(\begin{array}{c}
p(n) \\
n^{3}-1
\end{array}\right) \text {, where } p(n)=n^{3}+6 n^{2}-6 n+2
$$

The lower bound in Theorem 1 appeared in 1 and 2]; the upper bound was recently obtained in $[8$. The bounds in Theorem 1 are very loose as we see in Table 1 . Note that the exact number of the vertices of $\Omega_{4}$ is unknown to our knowledge at this time. For a general positive integer $n$, the determination of the number of vertices of $\Omega_{n}$ would be extremely difficult. 


\begin{tabular}{cccc}
\hline Case & lower & $f_{0}\left(\Omega_{n}\right)$ & upper \\
\hline$n=2$ & 1 & 2 & 21318 \\
$n=3$ & 2.37 & 66 & $\frac{1}{27}\left(\begin{array}{c}65 \\
26\end{array}\right)$ \\
$n=4$ & 25.63 & $?$ & $\frac{1}{64}\left(\begin{array}{c}138 \\
63\end{array}\right)$ \\
\hline
\end{tabular}

Table 1: Lower and upper bounds in Theorem 1

\section{A sharper upper bound}

We use the standard terminology in convex polytope theory such as $d$-dimensional polytope and $i$-faces of a polytope. A 0 -face is a vertex (or an extreme point) and a $(d-1)$-face is called a facet; $f_{i}$ denotes the number of $i$-faces. One may refer to texts [5] and [19] for the definitions of these terms. A fundamental question in the theory of convex polytope is the determination of the largest and the smallest numbers of $i$-faces, for instance, the vertices, of a polytope.

Proved by McMullen in 1970 [16], the Upper Bound Theorem (UBT) is one of the most important results in the combinatorial theory of polytopes. The UBT gives the maximum number of faces, say facets, of any $d$-polytope with a given number of vertices, and in a dual form, it gives the maximum number of vertices of any $d$-polytope with a given number of $k$-faces. Further, the UBT establishes that the asserted maximum numbers are achieved by cyclic polytopes (see, e.g., [19, p. 16 and p. 254]). As a consequence (see, e.g., [5, p. 90] and use duality), the number $f_{0}$ of vertices of a convex polytope of dimension $d$ with $f_{d-1}$ facets is bounded as follows:

$$
f_{0} \leq\left(\begin{array}{c}
f_{d-1}-\left\lfloor\frac{d+1}{2}\right\rfloor \\
f_{d-1}-d
\end{array}\right)+\left(\begin{array}{c}
f_{d-1}-\left\lfloor\frac{d+2}{2}\right\rfloor \\
f_{d-1}-d
\end{array}\right)
$$

Now we turn our attention to the number of vertices of the polytope $\Omega_{n}$ of $n \times n \times n$ stochastic tensors. Regarded as a subset of $\mathbb{R}^{n^{3}}, \Omega_{n}$ defined by (1)-(4) is the same as the set of all $x=\left(x_{i j k}\right) \in \mathbb{R}^{n^{3}}$ satisfying

$$
\begin{gathered}
x_{i j k} \geq 0, \quad 1 \leq i, j, k \leq n \\
\sum_{i=1}^{n} x_{i j k}=1, \quad 1 \leq j \leq n, 1 \leq k \leq n \\
\sum_{j=1}^{n} x_{i j k}=1, \quad 1 \leq i \leq n, 1 \leq k \leq n-1 \\
\sum_{k=1}^{n} x_{i j k}=1, \quad 1 \leq i \leq n-1,1 \leq j \leq n-1
\end{gathered}
$$


Observe that $n(2 n-1)+(n-1)^{2}=3 n^{2}-3 n+1$ independent line sum conditions are needed in defining $\Omega_{n}$ as a subset of $\mathbb{R}^{n^{3}}$. (In fact, the rank of the coefficient matrix of the linear equation system $(7)-(9)$ is $3 n^{2}-3 n+1$.) Since each linearly independent linear equation reduces the dimension by 1 , it follows that $\Omega_{n}$ as a polytope in $\mathbb{R}^{n^{3}}$ has dimension $d=n^{3}-\left(3 n^{2}-3 n+1\right)=$ $(n-1)^{3}$. Alternatively, it can be computed as follows: Of the $n^{3}$ variables, $n^{3}-3 n^{2}+3 n-1=(n-1)^{3}$ variables are free (equivalently, we can take the variables in the $(n-1) \times(n-1) \times(n-1)$ cube in the lower-front-left corner as free variables).

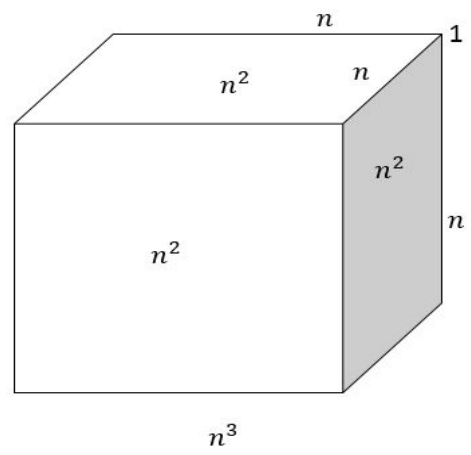

Figure 1: Computing the dimension by using the cube

As a polytope in $\mathbb{R}^{n^{3}}, \Omega_{n}$ is of dimension $d=(n-1)^{3}$ and has $f_{d-1}=n^{3}$ facets since each of the conditions in (6) yields a facet. By Carathéodory's Theorem, any $n \times n$ doubly stochastic matrix is a convex combination of at most $(n-1)^{2}+1$ permutation matrices. Likewise, any $n \times n \times n$ stochastic tensor is a convex combination of at most $(n-1)^{3}+1$ vertices (not necessarily permutation tensors).

Applying the above result (5) to $\Omega_{n}$, with $d=(n-1)^{3}$ and $f_{d-1}=n^{3}$, we arrive at a tighter bound for the number of vertices of $\Omega_{n}$.

Theorem 2 Let $f_{0}\left(\Omega_{n}\right)$ be the number of vertices of the polytope $\Omega_{n}$. Then

$$
f_{0}\left(\Omega_{n}\right) \leq\left(\begin{array}{c}
n^{3}-\left\lfloor\frac{(n-1)^{3}+1}{2}\right\rfloor \\
3 n^{2}-3 n+1
\end{array}\right)+\left(\begin{array}{c}
n^{3}-\left\lfloor\frac{(n-1)^{3}+2}{2}\right\rfloor \\
3 n^{2}-3 n+1
\end{array}\right)
$$

Proof. The polytope $\Omega_{n}$ is contained in an $(n-1)^{3}$-dimensional affine subspace of the $n^{3}$-dimensional space $\mathbb{R}^{n^{3}}$ determined by the linear equality constraints that the sum of each row and of each column be one. Within this affine subspace, it is defined by $n^{3}$ linear inequalities, one for each coordinate of the tensor (hypermatrix), specifying that the coordinate be non-negative. Therefore, it has exactly $n^{3}$ facets $F_{i j k}=\left\{x \in \Omega_{n} \mid x_{i j k}=0\right\}$, for $1 \leq i, j, k \leq n$. That is, $d=(n-1)^{3}$ and $f_{d-1}=n^{3}$ in (5). This completes the proof. 
We claim that the upper bound in Theorem 2 is better (tighter) than the one in Theorem 1 That is, we show the following inequality.

Proposition 3 Let $n \geq 2$ be a positive integer. Then

$$
\left(\begin{array}{c}
n^{3}-\left\lfloor\frac{(n-1)^{3}+1}{2}\right\rfloor \\
3 n^{2}-3 n^{2}+1
\end{array}\right)+\left(\begin{array}{c}
n^{3}-\left\lfloor\frac{(n-1)^{3}+2}{2}\right\rfloor \\
3 n^{2}-3 n+1
\end{array}\right)<\frac{1}{n^{3}}\left(\begin{array}{c}
n^{3}+6 n^{2}-6 n+2 \\
n^{3}-1
\end{array}\right)
$$

Proof. Direct computations for the cases $n=2,3,4,5$ show that the proposition holds. (In fact, for each case, the number on the right hand side is much larger than that on the left hand side.) Now we assume $n \geq 6$.

Let $a, b, k$ be positive integers such that $a \geq b$ and $k \geq 1$. Bear in mind the monotonicity properties that $\left(\begin{array}{l}a \\ b\end{array}\right) \leq\left(\begin{array}{c}a+k \\ b\end{array}\right)$ and that $\left(\begin{array}{c}a \\ b\end{array}\right) \leq\left(\begin{array}{c}a \\ b+k\end{array}\right)$ if $b+k \leq\left\lfloor\frac{a}{2}\right\rfloor$.

It follows that, for $n \geq 6$ (which is used for the last inequality),

$$
\begin{gathered}
\left(\begin{array}{c}
n^{3}-\left\lfloor\frac{(n-1)^{3}+1}{2}\right\rfloor \\
3 n^{2}-3 n+1
\end{array}\right)+\left(\begin{array}{c}
n^{3}-\left\lfloor\frac{(n-1)^{3}+2}{2}\right\rfloor \\
3 n^{2}-3 n^{2}+1
\end{array}\right) \\
<2\left(\begin{array}{c}
n^{3} \\
3 n^{2}-3 n+1
\end{array}\right)<2\left(\begin{array}{c}
n^{3} \\
3 n^{2}
\end{array}\right)
\end{gathered}
$$

On the other hand, for $k \geq 1$,

$$
\left(\begin{array}{c}
a+k \\
b+k
\end{array}\right)=\prod_{i=1}^{k} \frac{(a+i)}{(b+i)} \cdot\left(\begin{array}{c}
a \\
b
\end{array}\right)>\left(\frac{a+k}{b+k}\right)^{k} \cdot\left(\begin{array}{c}
a \\
b
\end{array}\right)
$$

Hence (as $n \geq 6$ ), we have $n^{3}+6 n^{2}-6 n+2 \geq 2\left(6 n^{2}-6 n+3\right)$ and

$$
\begin{aligned}
& \frac{1}{n^{3}}\left(\begin{array}{c}
n^{3}+6 n^{2}-6 n+2 \\
n^{3}-1
\end{array}\right) \\
& \quad=\frac{1}{n^{3}}\left(\begin{array}{c}
n^{3}+6 n^{2}-6 n+2 \\
6 n^{2}-6 n+3
\end{array}\right) \\
& \quad>\frac{1}{n^{3}}\left(\begin{array}{c}
n^{3}+6 n^{2}-6 n+2 \\
3 n^{2}+20
\end{array}\right) \quad(\text { by monotonicity) } \\
& \quad>\frac{1}{n^{3}}\left(\frac{n^{3}+6 n^{2}-6 n-18}{3 n^{2}+20}\right)^{20} \cdot\left(\begin{array}{c}
n^{3}+6 n^{2}-6 n-18 \\
3 n^{2}
\end{array}\right) \\
& \quad>\frac{1}{n^{3}}\left(\frac{n}{3}\right)^{20} \cdot\left(\begin{array}{c}
n^{3} \\
3 n^{2}
\end{array}\right) \quad(\text { by monotonicity) } \\
& \quad>2\left(\begin{array}{c}
n^{3} \\
3 n^{2}
\end{array}\right)
\end{aligned}
$$

This completes the proof.

It is stated in [15, p. 170] that $\Omega_{n}$ (i.e., $\Omega_{n}^{(2)}$ in [15]) has fewer than $n^{3 n^{2}}$ vertices. We show that our bound in Theorem 2 is tighter than this one also. 


\begin{tabular}{cccc}
\hline Case & $f_{0}\left(\Omega_{n}\right)$ & new up. bound & old up. bound \\
\hline$n=2$ & 2 & 2 & 21318 \\
$n=3$ & 66 & 10395 & $\frac{1}{27}\left(\begin{array}{c}65 \\
26\end{array}\right)$ \\
$n=4$ & $?$ & $2\left(\begin{array}{c}50 \\
37\end{array}\right)$ & $\frac{1}{64}\left(\begin{array}{c}138 \\
63\end{array}\right)$ \\
\hline
\end{tabular}

Table 2: Comparison of upper bounds in Theorems $1 \& 2$

Proposition 4 Let $n \geq 2$ be a positive integer. Then

$$
\left(\begin{array}{c}
n^{3}-\left\lfloor\frac{(n-1)^{3}+1}{2}\right\rfloor \\
3 n^{2}-3 n+1
\end{array}\right)+\left(\begin{array}{c}
n^{3}-\left\lfloor\frac{(n-1)^{3}+2}{2}\right\rfloor \\
3 n^{2}-3 n^{2}+1
\end{array}\right)<n^{3 n^{2}}
$$

Proof. Direct computations show that the assertion holds for $n=2,3, \ldots, 10$. Let $n \geq 11$. Then $\frac{e n^{2}}{3 n^{2}-3 n+1}<1$, where $e \approx 2.718$. Observe the fact that

$$
\left(\begin{array}{c}
m \\
k
\end{array}\right)<\left(\frac{m e}{k}\right)^{k} \text { for any positive integers } m \text { and } k, m \geq k
$$

(which follows from the Stirling's formula $\sqrt{2 \pi} k^{k+\frac{1}{2}} e^{-k}<k$ !). We have

$$
\begin{aligned}
& \left(\begin{array}{c}
n^{3}-\left\lfloor\frac{(n-1)^{3}+1}{2}\right\rfloor \\
3 n^{2}-3 n+1
\end{array}\right)+\left(\begin{array}{c}
n^{3}-\left\lfloor\frac{(n-1)^{3}+2}{2}\right\rfloor \\
3 n^{2}-3 n+1
\end{array}\right) \\
& \quad<2\left(\begin{array}{c}
n^{3} \\
3 n^{2}-3 n+1
\end{array}\right) \\
& \quad<2\left(\frac{e n^{3}}{3 n^{2}-3 n+1}\right)^{3 n^{2}-3 n+1} \\
& \quad=2\left(\frac{e n^{2}}{3 n^{2}-3 n+1}\right)^{3 n^{2}-3 n+1} \cdot n^{3 n^{2}-3 n+1} \\
& \quad<2 n^{3 n^{2}-3 n+1}<n^{3 n^{2}}
\end{aligned}
$$

Thus, we conclude that our new upper bound is tighter than the existing ones (to our knowledge). In fact, the left hand side is much smaller than the right hand side. For instance, for $n=10$, the quantity on the left is $2 \cdot\left(\begin{array}{c}635 \\ 271\end{array}\right) \approx$ $9.8 \times 10^{186}$, while on the right hand side, we have $10^{300}$.

\section{On the lower bound}

In the previous section, we saw that the upper bound for the number of vertices of $\Omega_{n}$ obtained by the McMullen Upper Bound Theorem (UBT) is better than 
the known ones. A natural and analogous question to ask is: how about the lower bound? In this section we obtain a lower bound through a lower bound theorem and compare the lower bound derived this way to the existing ones.

Let $L_{n}$ denote the number of $n \times n$ Latin squares. $L_{n}$ can be computed by

$$
\left.L_{n}=n ! \sum_{A \in B_{n}}(-1)^{\sigma_{0}(A)} \underset{n}{\operatorname{per} A}\right)
$$

where $B_{n}$ is the set of all 0-1 $n \times n$ matrices, $\sigma_{0}(A)$ is the number of zero entries in matrix $A$, and per $A$ is the permanent of matrix $A$; see Shao and Wei [18]. Note that $L_{n} \geq \frac{(n !)^{2 n}}{n^{n^{2}}}$ (see, e.g., [17, p. 162]).

Since every Latin square can be interpreted as a $0-1$ permutation tensor (see, e.g., [17, p. 159] or [9, Proposition 2.6]) and every $n \times n \times n$ 0-1 permutation tensor is an extreme point of $\Omega_{n}$, we have

$$
\frac{(n !)^{2 n}}{n^{n^{2}}} \leq L_{n} \leq f_{0}\left(\Omega_{n}\right)
$$

In contrast to the Upper Bound Theorem (UBT), the so-called Barnette Lower Bound Theorem (LBT) holds only for simplicial polytopes (see, e.g., 11, p. 166]). Note that the polytope of stochastic tensors is not simplicial in general. For example, when $n=3$, if $\Omega_{3}$ were simplicial, then according to Barnette's formula (see, e.g., [3] $f_{d-1} \geq(d-1) f_{0}-(d+1)(d-2)$, with $d=8, f_{d-1}=27$, $\Omega_{3}$ would have no more than 12 extreme points. This contradicts the fact that $\Omega_{3}$ has 66 vertices. Nevertheless, a lower bound for the number of vertices of a general $d$-polytope with $f_{d-1}$ facets is provided in [10, Theorem 1.4] (see also [11, Theorem 1.4])

$$
f_{0} \geq l_{0}^{d}\left(f_{d-1}\right)
$$

where

$$
l_{0}^{d}(x)=k \quad \text { if and only if } \quad u_{0}^{d}(k-1)<x \leq u_{0}^{d}(k)
$$

with $m=k-1$ and $k$ in

$$
u_{0}^{d}(m)=\left(\begin{array}{c}
m-\left\lfloor\frac{d}{2}\right\rfloor-1 \\
\left\lfloor\frac{d-1}{2}\right\rfloor
\end{array}\right)+\left(\begin{array}{c}
m-\left\lfloor\frac{d-1}{2}\right\rfloor-1 \\
\left\lfloor\frac{d}{2}\right\rfloor
\end{array}\right)
$$

For example, if $x=27$ and $d=8$, we want to find $k$ for which

$$
\left(\begin{array}{c}
k-6 \\
3
\end{array}\right)+\left(\begin{array}{c}
k-5 \\
4
\end{array}\right)<27 \leq\left(\begin{array}{c}
k-5 \\
3
\end{array}\right)+\left(\begin{array}{c}
k-4 \\
4
\end{array}\right)
$$

By a straightforward computation, we arrive at $k=11$. So, $l_{0}^{8}(27)=11$.

Theorem 5 Let $f_{0}\left(\Omega_{n}\right)$ be the number of vertices of the polytope $\Omega_{n}$. Then

$$
f_{0}\left(\Omega_{n}\right) \geq l_{0}^{(n-1)^{3}}\left(n^{3}\right)
$$

where $l_{0}^{(n-1)^{3}}\left(n^{3}\right)=k$ is such that (see (14) and (15))

$$
u_{0}^{(n-1)^{3}}(k-1)<n^{3} \leq u_{0}^{(n-1)^{3}}(k)
$$


Proof. Applying 13 with $d=(n-1)^{3}$ and $f_{d-1}=n^{3}$ yields immediately the lower bound (16).

In light of Theorems 1 and 2 on the upper bound, we would naturally propose

$$
l_{0}^{(n-1)^{3}}\left(n^{3}\right) \geq L_{n} \quad\left(\text { which is } \geq \frac{(n !)^{2 n}}{n^{n^{2}}}\right)
$$

It turns out that this is not the case in general; see the second part of Proposition 8. To compare the lower bounds, we first observe a fact that if $a$ and $b$ are positive integers such that $a \geq b+2$ and $b \geq 2$, then

$$
\left(\begin{array}{l}
a \\
b
\end{array}\right) \geq\left(\begin{array}{l}
a \\
2
\end{array}\right)
$$

This is because $\frac{a-b+r-2}{r} \geq 1$ when $a \geq b+2$ for $r=3,4, \ldots, b$, and

$$
\begin{aligned}
\left(\begin{array}{l}
a \\
b
\end{array}\right) & =\frac{a(a-1) \cdots(a-b+1)}{1 \cdot 2 \cdots b} \\
& =\left(\begin{array}{l}
a \\
2
\end{array}\right) \cdot \frac{a-b+1}{3} \cdot \frac{a-b+2}{4} \cdots \frac{a-b+r-2}{r} \cdots \frac{a-2}{b} \\
& \geq\left(\begin{array}{l}
a \\
2
\end{array}\right)
\end{aligned}
$$

Proposition 6 Let $n \geq 4$. If $k$ is a positive integer satisfying

$$
\begin{gathered}
\left(\begin{array}{c}
(k-1)-\left\lfloor\frac{(n-1)^{3}}{2}\right\rfloor-1 \\
\left\lfloor\frac{(n-1)^{3}-1}{2}\right\rfloor
\end{array}\right)+\left(\begin{array}{c}
(k-1)-\left\lfloor\frac{(n-1)^{3}-1}{2}\right\rfloor-1 \\
\left\lfloor\frac{(n-1)^{3}}{2}\right\rfloor
\end{array}\right) \\
\quad\left(\begin{array}{c}
k-\left\lfloor\frac{\left.(n-1)^{3}\right\rfloor}{2}\right\rfloor-1 \\
\left\lfloor\frac{(n-1)^{3}-1}{2}\right\rfloor
\end{array}\right)+\left(\begin{array}{c}
k-\left\lfloor\frac{(n-1)^{3}-1}{2}\right\rfloor-1 \\
\left\lfloor\frac{(n-1)^{3}}{2}\right\rfloor
\end{array}\right)
\end{gathered}
$$

then

$$
k=(n-1)^{3}+2
$$

Proof. Case 1. Suppose that $n$ is even. Then

$$
\left\lfloor\frac{(n-1)^{3}}{2}\right\rfloor=\frac{(n-1)^{3}-1}{2}, \quad\left\lfloor\frac{(n-1)^{3}-1}{2}\right\rfloor=\frac{(n-1)^{3}-1}{2}
$$

Let $p=\frac{(n-1)^{3}+3}{2}$. Then $p \geq 15$ (for $n \geq 4$ ). We write

$$
\left(\begin{array}{c}
(k-1)-\left\lfloor\frac{(n-1)^{3}}{2}\right\rfloor-1 \\
\left\lfloor\frac{(n-1)^{3}-1}{2}\right\rfloor
\end{array}\right)+\left(\begin{array}{c}
(k-1)-\left\lfloor\frac{(n-1)^{3}-1}{2}\right\rfloor-1 \\
\left\lfloor\frac{(n-1)^{3}}{2}\right\rfloor
\end{array}\right)=2\left(\begin{array}{c}
k-p \\
p-2
\end{array}\right)
$$

and

$$
\left(\begin{array}{c}
k-\left\lfloor\frac{(n-1)^{3}}{2}\right\rfloor-1 \\
\left\lfloor\frac{(n-1)^{3}-1}{2}\right\rfloor
\end{array}\right)+\left(\begin{array}{c}
k-\left\lfloor\frac{(n-1)^{3}-1}{2}\right\rfloor-1 \\
\left\lfloor\frac{(n-1)^{3}}{2}\right\rfloor
\end{array}\right)=2\left(\begin{array}{c}
k-p+1 \\
p-2
\end{array}\right)
$$


The inequalities in the proposition become

$$
2\left(\begin{array}{c}
k-p \\
p-2
\end{array}\right)<n^{3} \leq 2\left(\begin{array}{c}
k-p+1 \\
p-2
\end{array}\right)
$$

By (18), $k-p+1 \geq p-2+1$, i.e., $k \geq 2 p-2$. If $k=2 p-2$, then 18 becomes

$$
2<n^{3} \leq 2(p-1)=(n-1)^{3}+1
$$

which is impossible for $n>1$. Hence, $k \geq 2 p-1$. We claim $k=2 p-1$. Suppose otherwise that $k \geq 2 p$. Then $k-p \geq p-2+2$ and $p-2 \geq 2$. By (17) we have

$$
\begin{aligned}
2\left(\begin{array}{c}
k-p \\
p-2
\end{array}\right) & \geq 2\left(\begin{array}{c}
k-p \\
2
\end{array}\right)=(k-p)(k-p-1) \geq(2 p-p)(2 p-p-1) \\
& =\frac{\left((n-1)^{3}+3\right) \cdot\left((n-1)^{3}+1\right)}{4}>\frac{(n-1)^{6}}{4} \geq n^{3}
\end{aligned}
$$

contradicting (18). So, $k=2 p-1=(n-1)^{3}+2$. With such $k, 18$ becomes

$$
(n-1)^{3}+1<n^{3} \leq\left(\frac{(n-1)^{3}+1}{2}\right)\left(\frac{(n-1)^{3}+3}{2}\right)
$$

which holds for $n \geq 4$ by direct verifications.

Case 2. Suppose that $n \geq 5$ is odd. Then

$$
\left\lfloor\frac{(n-1)^{3}}{2}\right\rfloor=\frac{(n-1)^{3}}{2},\left\lfloor\frac{(n-1)^{3}-1}{2}\right\rfloor=\frac{(n-1)^{3}-2}{2}
$$

Let $r=\frac{(n-1)^{3}+4}{2}=\frac{(n-1)^{3}}{2}+2 \geq 5$. The inequalities in the proposition becomes

$$
\left(\begin{array}{c}
k-r \\
r-3
\end{array}\right)+\left(\begin{array}{c}
k-r+1 \\
r-2
\end{array}\right)<n^{3} \leq\left(\begin{array}{c}
k-r+1 \\
r-3
\end{array}\right)+\left(\begin{array}{c}
k-r+2 \\
r-2
\end{array}\right)
$$

We claim that $k=2 r-2=(n-1)^{2}+2$. To show this, we draw contradictions for both cases (i) $k<2 r-2$ and (ii) $k>2 r-2$.

(i) If $k \leq 2 r-3$, i.e., $k-r+1 \leq(r-3)+1, k-r+2 \leq(r-2)+1$, then

$$
\begin{aligned}
\left(\begin{array}{c}
k-r+1 \\
r-3
\end{array}\right)+\left(\begin{array}{c}
k-r+2 \\
r-2
\end{array}\right) & \leq(k-r+1)+(k-r+2) \\
& =2 k-2 r+3 \\
& \leq 2(2 r-3)-2 r+3 \\
& =2 r-3=(n-1)^{3}+1<n^{3}
\end{aligned}
$$

contradicting (19).

(ii) If $k \geq 2 r-1$, then $k-r \geq(r-3)+2, k-r+1 \geq(r-2)+2$. By (17), we have

$$
\begin{aligned}
\left(\begin{array}{c}
k-r \\
r-3
\end{array}\right)+\left(\begin{array}{c}
k-r+1 \\
r-2
\end{array}\right) & \geq\left(\begin{array}{c}
k-r \\
2
\end{array}\right)+\left(\begin{array}{c}
k-r+1 \\
2
\end{array}\right) \\
& =(k-r)^{2} \geq(r-1)^{2} \\
& >\frac{(n-1)^{6}}{4}>n^{3}
\end{aligned}
$$


also contradicting 19 . It follows that $k=2 r-2=(n-1)^{3}+2$.

Corollary 7 Let $l_{0}^{(n-1)^{3}}\left(n^{3}\right)$ be defined by (14) and 15). Then

$$
l_{0}^{(n-1)^{3}}\left(n^{3}\right)= \begin{cases}11, & \text { for } n=3 \\ (n-1)^{3}+2, & \text { for } n \geq 4\end{cases}
$$

Proposition 8 Let $l_{0}^{(n-1)^{3}}\left(n^{3}\right)$ be defined by (14) and (15).

(1). If $n=3$ or $n=4$, then

$$
l_{0}^{(n-1)^{3}}\left(n^{3}\right)>\frac{(n !)^{2 n}}{n^{n^{2}}}
$$

(2). If $n \geq 5$, then

$$
l_{0}^{(n-1)^{3}}\left(n^{3}\right)<\frac{(n !)^{2 n}}{n^{n^{2}}}
$$

Proof. We compute as follows:

$$
\begin{array}{ll}
n=3: & \frac{(n !)^{2 n}}{n^{n^{2}}}=\frac{64}{27}=2.37<l_{0}^{8}(27)=11 \\
n=4: & \frac{(n !)^{2 n}}{n^{n^{2}}}=\frac{6561}{256}=25.6<l_{0}^{27}(64)=29 \\
n=5: & \frac{(n !)^{2 n}}{n^{n^{2}}}=\left(\frac{24^{2}}{5^{3}}\right)^{5}=4.6^{5}>l_{0}^{64}(125)=66 \\
n=6: & \frac{(n !)^{2 n}}{n^{n^{2}}}=\left(\frac{20}{6}\right)^{12}>3^{12}>l_{0}^{125}(216)=127 \\
n=7: & \frac{(n !)^{2 n}}{n^{n^{2}}}=\frac{24^{14} 30^{14}}{7^{35}}=\left(\frac{24}{7}\right)^{14} \cdot\left(\frac{30}{7}\right)^{14} \cdot \frac{1}{7^{7}}>l_{0}^{216}(343)=218
\end{array}
$$

For $n \geq 8$, using the fact that $\sqrt{2 n \pi}\left(\frac{n}{e}\right)^{n}<n$ !, we have

$$
\begin{aligned}
(n-1)^{3}+2 & \leq(2 n \pi)^{n} \\
& \leq(2 n \pi)^{n} \cdot\left(\frac{n}{e^{2}}\right)^{n^{2}} \\
& =\frac{\left(\sqrt{2 n \pi}\left(\frac{n}{e}\right)^{n}\right)^{2 n}}{n^{n^{2}}} \\
& <\frac{(n !)^{2 n}}{n^{n^{2}}}
\end{aligned}
$$

We thus conclude that the lower bound for the number of the vertices of $\Omega_{n}$ through the lower bound theorem for a general polytope is looser than the existing ones when $n \geq 5$. 


\section{Discussions}

We have seen that the lower and upper bounds for the number of vertices of $\Omega_{n}$

$$
L_{n} \leq f_{0}\left(\Omega_{n}\right) \leq U_{n}
$$

where $L_{n}$ is the number of $n \times n$ Latin squares and $U_{n}$ (see Theorem 2 ) is

$$
U_{n}=\left(\begin{array}{c}
n^{3}-\left\lfloor\frac{(n-1)^{3}+1}{2}\right\rfloor \\
3 n^{2}-3 n+1
\end{array}\right)+\left(\begin{array}{c}
n^{3}-\left\lfloor\frac{(n-1)^{3}+2}{2}\right\rfloor \\
3 n^{2}-3 n+1
\end{array}\right)
$$

The bounds depending solely and explicitly on $n$ in 20 are the "best" ones to our knowledge. However, as Table 2 (for the case of upper bound) and numerical computations show that these bounds are still very loose. This is also seen in the proofs of the present results. Better estimates have been called for (see [15, Section 4]). Determination of the exact number of vertices (or even harder, $d$-faces) of a general polytope is an extremely difficult problem. The bounds we have obtained in fact are good for all polytopes of dimension $(n-1)^{3}$ with $n^{3}$ facets. A better estimate of the number of vertices specifically for the polytopes of stochastic arrays or Birkhoff type or $\Omega_{n}$ in particular is desired.

In their paper [15] on the vertices of $d$-dimensional Birkhoff polytope, Linial and Luria use Latin squares and present a lower bound for the number of vertices of $\Omega_{n}$ (i.e., $\Omega_{n}^{(2)}$ in [15, Theorem 1.5]) with indeterminants $o(1)$ :

Theorem 9 (Linial and Luria [15]) The polytope $\Omega_{n}$ has at least $L_{n}^{\frac{3}{2}-o(1)}$ vertices and $L_{n}=\left((1+o(1)) \frac{n}{e^{2}}\right)^{n^{2}}$. (Note: the two o(1)s may be different.)

Thus, for the lower bound, we can write $L_{n}^{\frac{3}{2}-o(1)} \leq f_{0}\left(\Omega_{n}\right)$. The shortcoming of this lower bound is that it contains the indeterminants $o(1) \mathrm{s}$; it gives an estimate but not the exact value for given $n$. Note that it is known [17, p. 162, Theorem 17.3] that $L_{n}^{1 / n^{2}} \sim e^{-2} n$ as $n \rightarrow \infty$.

Acknowledgement. Fuzhen Zhang is thankful to Richard Brualdi for drawing reference [15] to his attention. The authors thank Chi-Kwong Li for his comments in the early stage of the project. Fuzhen Zhang's work was partially supported by National Natural Science Foundation of China (NNSFC) No. 11571220 via Shanghai University. Xiao-Dong Zhang's work was partially supported by NNSFC No. 11531001, No. 11271256 and NNSFC-ISF Research Program No. 11561141001.

\section{References}

[1] Ahmed M. Algebraic Combinatorics of Magic Squares, University of Califorina Davis, Ph.D. Thesis, 2004. 
[2] Ahmed M, De Loera J, Hemmecke R. Polyhedral Cones of Magic Cubes and Squares, in Discrete and Computational Geometry Algorithms and Combinatorics, Vol. 25, pp. 25-41 (eds B. Aronov et al), 2003, Springer.

[3] Barnette D. A proof of the lower bound conjecture for convex polytopes, Pacific J. Math. 46 (1973), no. 2, 349-354.

[4] Barvinok A. A Course in Convexity, Graduate Studies in Mathematics, 54. American Mathematical Society, Providence, RI, 2002.

[5] Brondsted A. An Introduction to Convex Polytopes, Springer, 1983.

[6] Brualdi RA, Csima J. Stochastic patterns, J. Combin. Theory Ser. A 19 (1975) $1-12$.

[7] Brualdi RA, Csima J. Extremal plane stochastic matrices of dimension three, Linear Algebra Appl. 11 (1975) 105-133.

[8] Chang H, Paksoy VE, Zhang F. Polytopes of Stochastic Tensors, Annals of Functional Analysis, Vol. 7, Number 3 (2016), 386-393.

[9] Cui L-B, Li W, Ng MK. Birkhoff-von Neumann Theorem for Multistochastic Tensors, SIAM. J. Matrix Anal. \& Appl. 35 (2014) 956-973.

[10] Deza A. On lower bound for general convex polytopes. Math. Japon. 40 (1994), no. $2,371-380$.

[11] Deza A, Fukuda K. McMullen's conditions and some lower bounds for general convex polytopes, Geom. Dedicata 52 (1994), no. 2, 165-173.

[12] Fischer P, Swart ER. Three dimensional line stochastic matrices and extreme points, Linear Algebra Appl. 69 (1985) 179-203.

[13] Gromova MB. The Birkhoff-von Neumann theorem for polystochastic matrices [translation of Operations research and statistical simulation, No. 2 (Russian), 315, 149, Izdat. Leningrad. Univ., Leningrad, 1974]. Selected translations. Selecta Math. Soviet. 11 (1992), no. 2, 145-158.

[14] Jurkat WB, Ryser HJ. Extremal Configurations and Decomposition Theorems. I, J. Algebra 8 (1968) 194-222.

[15] Linial N, Luria Z. On the vertices of the d-dimensional Birkhoff polytope, Discrete Comput. Geom. 51 (2014), no. 1, 161-170.

[16] McMullen P. The maximum numbers of faces of a convex polytope, Mathematika 17 (1970) 179-184.

[17] van Lint JH, Wilson RM. A Course in Combinatorics, Cambridge University Press, 1992.

[18] Shao J-Y, Wei W-D. A formula for the number of Latin squares, Discrete Math., Vol. 110 (1992), Issues 1-3, 293-296.

[19] Ziegler GM. Lectures on Polytopes, Springer, 1995. 